\title{
Can You Cover Your Shadows?
}

\author{
H. E. Debrunner and P. Mani-Levitska \\ Mathematisches Institut, Universität Bern, Sidlerstrasse 5, 3012 Bern, Switzerland
}

\begin{abstract}
A subset $X$ of the $d$-dimensional Euclidean space $\mathbb{R}^{d}$ can cover its shadows in $\mathbf{R}^{d}$, if every orthogonal projection of $X$ onto a $(d-1)$-dimensional linear subspace of $\mathbb{R}^{d}$ is contained in some congruent copy of $X$. Whereas every two-dimensional convex disc $C \subset \mathbf{R}^{d}$ has this property, no $(d-1)$-polytope does, provided that $d \geq 4$.
\end{abstract}

\section{Introduction}

This contribution originates in a question which has been posed independently by Pach [8] and Zalgaller. With the terminology of Definition 2 below, they asked whether every two-dimensional convex disc can cover its shadows in the ordinary space $\mathbb{R}^{3}$. Janos Pach told us that he likes to put this question in a more dynamical context: If a convex body (say, a stone) can be thrust through a convex hole in the wall by a linear motion (without twisting) then can this be done by a movement perpendicular to the wall? The answer to this question is positive and, again, of twofold origin. One solution is due to Kowaljov [5]. Let us give a brief outline of his argument. With the notation of Definition 3 below, it is enough to prove that every strictly convex disc $F \subset \mathbf{R}^{2}$, whose boundary curve is twice continuously differentiable, can cover its small contractions in direction $e_{2}=(0,1)$. We say that $Y \subset \mathbb{R}^{2}$ arises from the convex disc $X \subset \mathbf{R}^{2}$ by a skew expansion, if there are two parallel supporting lines $G_{1}, G_{2}$ of $X$ and points $p_{i} \in X \cap G_{i}$, $i \in\{1,2\}, r \in G_{1} \backslash\left\{p_{1}\right\}, q \in$ relintconv $\left\{p_{1}, r\right\}$, such that the affine map $f$ : $\mathbb{R}^{2} \rightarrow \mathbf{R}^{2}$ satisfying $f\left(p_{i}\right)=p_{i}, i \in\{1,2\}, f(q)=r$, carries $X$ onto $Y$. Given a strictly convex disc $F \subset \mathbf{R}^{2}$ as above and a disc $G=D\left(\lambda, e_{2}\right)[F]$ with $\lambda<1$ and sufficiently close to 1 , then $F$ arises from $G$ by some composition of at most two skew expansions, and motions. This statement, whose proof requires some ingenious analytic arguments, is the main step in Kowaljov's construction. Our solution, presented in Section 6, studies polygonal, rather than smooth, approximations, and then uses the passage to infinitesimals, together with Helly's 
theorem, to reduce the general case to that of arbitrary quadrangles. The fact that every convex quadrangle can cover its shadows in $\mathbf{R}^{3}$, which presents the only computational difficulty in this approach, has been known to Pach for quite some time. We would be happy to learn about a more elegant proof than the one of Section 4 below. In higher dimensions the situation changes radically: No convex $d$-polytope, with $d \geq 3$, can cover its shadows in $\mathbf{R}^{d+1}$. This leads to several open questions, some of which we have collected in Section 7.

Our basic geometric terminology follows Grünbaum's book [4], with a few small modifications. So we denote by $e_{i}$ the $i$ th vector in the standard basis of $\mathbf{R}^{d}$, whose $i$ th coordinate equals one, while all of its other coordinates vanish. When there is no danger of confusion we also write $x_{i}$ for the $i$ th coordinate of $x \in \mathbf{R}^{d}$, hence $x_{i}=\left\langle x, e_{i}\right\rangle$. It will often be convenient to identify $\mathbb{R}^{k}, k \leq d$, with the subspace lin $\left\{e_{1}, \ldots, e_{k}\right\}$ of $\mathbb{R}^{d}$. The Euclidean unit ball and unit sphere in $\mathbf{R}^{d}$ are denoted by $B^{d}=\left\{x \in \mathbf{R}^{d}:\|x\| \leq 1\right\}$ and $S^{d-1}=\left\{x \in \mathbb{R}^{d}:\|x\|=1\right\}$. By $x^{\perp}$ we understand the orthogonal subspace to $x \in \mathbf{R}^{d}, x^{\perp}=\left\{y \in \mathbf{R}^{d}:\langle x, y\rangle=0\right\}$. $\Gamma(d, k), 0 \leq k \leq d$, stands for the Grassmann manifold of all $k$-dimensional linear subspaces of $\mathbb{R}^{d}$. We consider the group isom $\left(\mathbf{R}^{d}\right)$ of all isometries of $\mathbf{R}^{d}$, its normal subgroup isom ${ }_{+}\left(\mathbf{R}^{d}\right)$ of all orientation preserving isometries, also called proper motions, of $\mathbf{R}^{d}$, as well as some other subgroups, such as the orthogonal group $O(d)$ and the rotation group $\mathrm{SO}(d)=\mathrm{O}(d) \cap$ isom $_{+}\left(\mathbf{R}^{d}\right) .(d \times d)$-matrices act upon $\mathbf{R}^{d}$ as linear operators in the usual way, the matrix $M=\left(M_{i j}\right)$ transforms $x$ to the vector $M x$, given by $(M x)_{i}=\sum_{j=1}^{d} M_{i j} x_{j}$. The norm of $M$ is defined by $\|M\|=\left(\sum_{i, j=1}^{d} M_{i j}^{2}\right)^{1 / 2}$. We write $d(x, A)$ for the distance between $x \in \mathbf{R}^{d}$ and $A \subset \mathbf{R}^{d}, A \neq \varnothing$. If $A$ is closed and convex, there exists a unique point $y$ in $A$, the nearest point $\nu_{A}(x)$, satisfying $\|y-x\|=d(x, A)$. The basic properties of the nearest point map $\nu_{A}: \mathbb{R}^{d} \rightarrow A$ are discussed in [6]. By $\mathscr{C}_{d}$ we denote the space of all nonempty compact, convex subsets of $\mathbf{R}^{d}$, with the Hausdorff distance $\delta$. A convex body in $\mathbf{R}^{d}$ is a $d$-dimensional element of $\mathscr{C}_{d}$. The vector $n \in S^{d-1}$ is called an outer normal of $C \in \mathscr{C}_{d}$ at the point $x \in \operatorname{bd}(\mathcal{C})$, if $\langle n, x\rangle \geq\langle n, y\rangle$ for every $y \in C$. If $P \in \mathscr{C}_{d}$ is a polytope, we denote by $\partial P$ the set of its proper faces. We call $n \in S^{d-1}$ an outer normal of $P$ at the face $F \in \partial P$, if $\langle n, x\rangle \geq\langle n, y\rangle$ for some $x \in \operatorname{relint}(F)$ and every $y \in P$. In this case $x+n^{\perp}$ is a supporting hyperplane of $P$, containing $F$. The following definitions lead to our main theme. If $X_{1}=\left(X_{1}, d_{1}\right)$ and $X_{2}=\left(X_{2}, d_{2}\right)$ are metric spaces we say that a map $f$ : $X_{1} \rightarrow X_{2}$ is a general contraction, if $d_{2}(f p, f q) \leq d_{1}(p, q)$ for all pairs $p, q$. The word contraction, without further attribute, is reserved here for those maps $f$ : $\mathbb{R}^{d} \rightarrow \mathbf{R}^{d}$ which are linear, have nonnegative determinant, and are general contractions with respect to the standard Euclidean metric of $\mathbf{R}^{d}$. The directional contractions $D(u, \lambda), u \in S^{d-1}, 0 \leq \lambda \leq 1$, given by $D(u, \lambda)[x]=x+(\lambda-$ $1)\langle x, u\rangle u$, will receive our special attention. They connect the identity map $D(u, 1)$ to the orthogonal projection $\pi_{u}=D(u, 0)$ onto the $(d-1)$-dimensional subspace $u^{\perp}$. By abuse of language we call $f[A]$ a contraction of $A$, if $f$ itself is a contraction in the above sense. For real numbers $a$ and $b>a$ we use the abbreviations $] a, b[=\{x \in \mathbb{R}: a<x<b\}] a, b,]=] a, b[\cup\{b\}$, $[a, b[=] a, b[\cup\{a\},[a, b]=] a, b] \cup[a, b[$.

Definition 1. The set $A \subset \mathbb{R}^{d}$ can cover $B \subset \mathbf{R}^{d}$, if there exists a proper motion $r \in$ isom $_{+}\left(\mathbf{R}^{d}\right)$ such that $B \subset r[A]$. 
Definition 2. $A \subset \mathbb{R}^{d}$ can cover its shadows in $R^{d}$, if $A$ can cover $\pi_{u}[A]=$ $D(u, 0)[A]$, for every $u \in S^{d-1}$.

Definition 3. $A \subset \mathbf{R}^{d}$ can cover its contractions, if $A$ can cover $f[A]$, whenever $f: \mathbf{R}^{d} \rightarrow \mathbf{R}^{d}$ is a contraction. $A$ can cover its contractions in direction $u \in S^{d-1}$, if $A$ can cover $D(u, \lambda)[A]$, for every $\lambda \in[0,1]$. $A$ can cover its small contractions in direction $u$, if there is some number $\left.\lambda_{0} \in\right] 0,1[$ such that $A$ can cover $D(u, \lambda)[A]$, for every $\lambda \in\left[\lambda_{0}, 1\right]$.

A considerable part of our studies involves local centers and local axes.

Definition 4. Consider a set $A \subset \mathbf{R}^{d}$, containing the origin $o$ of $\mathbf{R}^{d}$ in its interior. $o$ is a local center for $A$, if we can associate to every pair $(u, N), u \in S^{d-1}, N$ some neighborhood of the identity in $O(d)$, a number $\left.\lambda_{0} \in\right] 0,1[$ and a map $\lambda \leftrightarrow r_{\lambda}$ from $\left[\lambda_{0}, 1\right]$ into $N$, such that $D(u, \lambda)[A] \subset r_{\lambda}[A]$ for every $\lambda \in\left[\lambda_{0}, 1\right]$.

This means that small directional contractions of $A$ can be covered by small rotations.

Definition 5. A line $G=\operatorname{lin}\{g\} \in \Gamma(d, 1)$ is a local axis for $A \subset \mathbf{R}^{d}$, if, given any $u \in g^{\perp} \cap S^{d-1}$ and any neighborhood $N$ of the identity in $O(d)$, we can find $\left.\lambda_{0} \in\right] 0,1\left[\right.$ and a map $\lambda \mapsto r_{\lambda}$ from $\left[\lambda_{0}, 1\right]$ into $N$, such that $r_{\lambda}(x)=x$ for every $x \in G$ and $D(u, \lambda)[A] \subset r_{\lambda}[A]$ for all $\lambda \in\left[\lambda_{0}, 1\right]$.

\section{Simple Facts}

The notions of the preceding section are not independent from one another. If $X \subset \mathbb{R}^{d}$ can cover its contractions, then it can trivially cover its shadows in $\mathbf{R}^{d}$. A few slightly less obvious facts are collected in the next two lemmas.

\section{Lemma 1}

If $X \subset \mathbf{R}^{d}$ can cover $Y \subset \mathbf{R}^{d}$, and $Y$ can cover $Z \subset \mathbf{R}^{d}$, then $X$ can cover $Z$.

If $\left(X_{n}\right)_{n \in \mathrm{N}}$ and $\left(Y_{n}\right)_{n \in \mathbf{N}}$ are converging sequences in $\mathscr{C}_{d}$, such that $X_{n}$ can cover $Y_{n}$, for every $n \in \mathbb{N}$, then $\lim _{n \rightarrow \infty} X_{n} \in \mathscr{C}_{d}$ can cover $\lim _{n \rightarrow \infty} Y_{n} \in \mathscr{C}_{d}$.

Lemma 1 is trivial: just remember that isom ${ }_{+}\left(\mathbf{R}^{d}\right)$ is a locally compact group, and that the elements of $\mathscr{C}_{d}$ are all compact.

\section{Lemma 2}

Consider $X \subset \mathbf{R}^{d} \subset \mathbf{R}^{n}$, with $d<n . X$ can cover its shadows in $\mathbf{R}^{n}$ if, and only if, it can cover every contraction $D(u, \lambda)[X], u \in S^{d-1}$ $\lambda \in[0,1]$ in $\mathbb{R}^{d}$.

Assume that $u \in S^{d-1}$ and $\mathscr{F} \subset \mathscr{C}_{d}$ are such that $D(u, \lambda)[X] \in \mathscr{F}$, for every $X \in \mathscr{F}$ and $\lambda \in] 0,1]$. If each element of $\mathscr{F}$ can cover its small contractions in direction $u$, then each element of $\mathscr{F}$ can cover its contractions in direction $u$. 
Assume that $\mathscr{G} \subset \mathscr{C}_{d}$ contains, together with each set $X$, every regular affine image of $X$. If there exists a direction $u \in S^{d-1}$ such that each $X \in \mathscr{G}$ can cover its small contractions in direction $u$, then each $X \in \mathscr{G}$ can cover its contractions.

\section{Proof}

Ad (3). Just observe that every directional contraction $D(u, \lambda)[X], u \in S^{d-1}$, $\lambda \in[0,1]$, of $X \subset \mathbb{R}^{d} \subset \mathbf{R}^{n}, d<n$, is congruent to the orthogonal projection of $X$ onto some $d$-dimensional linear subspace of $\mathbf{R}^{n}$, and vice versa.

Ad (4). Set, for $X \in \mathscr{F}, V_{X}=\{\mu \in[0,1]: X$ can cover $D(u, \lambda)[X]$, for all $\lambda \in[\mu, 1]\}$. We derive from (2) that $V_{X}$ is compact, hence $V_{X}=[\lambda, 1]$, for some $\lambda \geq 0$. In the case $\lambda>0$ we have $Y=D(u, \lambda)[X] \in \mathscr{F}$, consequently $Y$ can cover $D(u, \mu)[Y]$ for each $\mu \in\left[\mu_{0}, 1\right]$ with suitable $\left.\mu_{0} \in\right] 0,1[$. Now $X$ can cover $Y$; so, by (1), $X$ can cover $D(u, \mu)[Y]=D(u, \lambda \mu)[X]$, contradicting the definition of $\lambda$. Hence $\lambda=0$, and (4) follows.

Ad (5). Because of (4), every $Y \in \mathscr{G}$ can cover its contractions in direction $u$. Given $X \in \mathscr{G}$ and $(v, \lambda) \in S^{d-1} \times[0,1]$, we choose $r \in \mathrm{SO}(d)$ such that $r(u)=v$. Setting $Y=r^{-1}[X] \in \mathscr{G}$, we obtain a proper motion $s$ of $\mathbb{R}^{d}$ satisfying $D(u, \lambda)[Y]$ $\subset s[Y]$. A straightforward calculation shows that $D(v, \lambda)[X] \subset r s r^{-1}[X]$. This means that every $X \in \mathscr{G}$ can cover all its directional contractions. If $f: \mathbb{R}^{d} \rightarrow \mathbf{R}^{d}$ is an arbitrary nondegenerate contraction, we find an orthonormal basis $\left(v_{1}, \ldots, v_{d}\right)$ of $\mathbf{R}^{d}$, numbers $\left.\left.\lambda_{1} \in\right] 0,1\right], 1 \leq i \leq d$, and a rotation $r \in \operatorname{SO}(d)$, such that $f=$ $r \circ D\left(v_{d}, \lambda_{d}\right) \circ \cdots \circ D\left(v_{1}, \lambda_{1}\right)$. For a proof see [1, p. 14ff], with slight adaptations. Let us define the sets $X_{i} \in \mathscr{G}, 0 \leq i \leq d$, inductively, by $X_{0}=X, X_{i}=$ $D\left(v_{i}, \lambda_{i}\right)\left[X_{i-1}\right], 1 \leq i \leq d$. We have already established that $X_{i}$ can cover $X_{i+1}$, $0 \leq i \leq d-1$, and, with the aid of (1), conclude that $X$ can cover $f[X]$. If the contraction $f$ is degenerate, we use (2), together with the fact that $f$ may be approximated by nondegenerate contractions, to obtain some proper motion $r$ with $f[X] \subset r[X]$. This completes our proof of (5).

The following two lemmas show that each convex set, which can cover its contractions, gives rise to a whole family of sets with the same property.

Lemma 3. If $C \in \mathscr{C}_{d}$ can cover its contractions, then every parallel set $C+\rho B^{d}$, $\rho \geq 0$ in $\mathbf{R}^{d}$ can also cover its contractions.

Proof. In order to show that $D=C+\rho B^{d}, \rho \geq 0$, can cover its contractions, consider any linear map $f: \mathbf{R}^{d} \rightarrow \mathbf{R}^{d}$ with $\operatorname{Lipschitz}$ constant $\operatorname{Lip}(f) \leq 1$. By definition, $f[C] \subset r[C]$, for some $r \in$ isom $_{+}\left(\mathbf{R}^{d}\right)$. Every point $p \in D$ can be written as a sum $p=c+x$ for some $c \in C$ and some $x \in \mathbf{R}^{d}$ with $\|x\| \leq \rho$. We have $f(p)=f(c)+f(x)$, hence $d(f(p), f(c))=\|f(p)-f(c)\|=\|f(x)\| \leq \rho$. It follows that $f[D] \subset f[C]+\rho B^{d} \subset r[C]+\rho B^{d}=r\left[C+\rho B^{d}\right]$, as stated in our lemma.

Lemma 4. Assume that the d-dimensional compact convex set $C \subset \mathbf{R}^{d}$ can cover its contractions. Then $C-C$ can cover its contractions and the origin is a local center for $C-C$. 
Proof. To each contraction $f: \mathbf{R}^{d} \rightarrow \mathbf{R}^{d}$ we can associate elements $r$ of $\mathrm{SO}(d)$ and $t \in \mathbf{R}^{d}$, such that $f[C] \subset r[C]+t$. Hence $f[-C]=-f[C] \subset-(r[C]+t)=$ $r[-C]-t$. Because $f$ and $r$ are linear maps, this implies

$$
\begin{aligned}
f[C-C] & =f[C]+f[-C] \subset r[C]+t+r[-C]-t \\
& =r[C]+r[-C]=r[C-C] .
\end{aligned}
$$

It remains to show that the origin $o$ is not only a center of symmetry, but also a local center, for the convex body $S=C-C$. Given $u \in S^{d-1}$ and an open neighbourhood $N$ of 1 in $\operatorname{SO}(d)$, the above reasoning implies that $R(\lambda)=\{r \in$ $\mathrm{SO}(d): D(u, \lambda)[S] \subset r[S]\}$ is not empty, for any $\lambda \in[0,1]$. We want to prove that $W=\{\lambda \in[0,1]: R(\lambda) \cap N=\varnothing\}$ is bounded away from 1. Otherwise we obtain a sequence $\left(\lambda_{l}\right)_{i \in \mathbb{N}}$ in $W$, converging to 1 , and a sequence $\left(r_{t}\right)_{i \in \mathbb{N}}$ in $\mathrm{SO}(d)$, converging to some $r \in \mathrm{SO}(d)$, such that $r_{i} \in R\left(\lambda_{i}\right) i \in \mathbb{N}$.

Obviously $S=\lim _{\imath \rightarrow \infty} D\left(u, \lambda_{1}\right)[S] \subset \lim _{i \rightarrow \infty} r_{i}[S]=r[S]$, and $S \in \mathscr{C}_{d}$ implies $S=r[S]$.

We conclude that $D\left(u, \lambda_{i}\right)[S] \subset r_{i}[S]=r_{i} r^{-1}[S]$, for every $i \in \mathbf{N}$. But $r_{i} r^{-1}$ belongs to $N$, for sufficiently large $i$, which contradicts the fact that every $\lambda_{\text {, lies }}$ in $W$. Lemma 4 is established.

\section{A Sufficient and a Necessary Condition}

The requirements for a convex body $C \subset \mathbf{R}^{d}$, to cover its contractions, involve the Lie group isom $+\left(\mathbf{R}^{d}\right)$. It is convenient to pass to the corresponding conditions involving infinitesimal motions, that is, elements of the Lie algebra of isom ${ }_{+}\left(\mathbf{R}^{d}\right)$. The book [1] contains all the necessary background.

Lemma 5. Let $P$ be a d-dimensional convex polytope in $\mathbf{R}^{d}$, and $u \in S^{d-1}$ a unit vector. If there exist a skew symmetric $(d \times d)$-matrix $M$ and a vector $t \in \mathbb{R}^{d}$ such that, for every vertex $x$ of $P$ and every outer normal $n$ of $P$ at $x$,

$$
\langle M x+t-\langle x, u\rangle u, n\rangle<0
$$

then $P$ can cover its small contractions in direction $u$.

Proof. Denote by $X$ the $d^{2}$-dimensional vector space of all real $(d \times d)$-matrices and by $S$ its linear subspace, consisting of the skew symmetric matrices. If we identify $\mathrm{O}(d)$ with a $\left(\begin{array}{l}d \\ 2\end{array}\right)$-dimensional smooth submanifold of $X$, then $S$ can be considered as the tangent space of $\mathrm{O}(d)$ at the identity, $S=T_{1} \mathrm{O}(d)$. Let us write $\exp _{0}: S \rightarrow \mathrm{O}(d)$ for the exponential map. Similarly, the group $D_{u}$ of all positive dilatations in direction $u \in S^{d-1}$ has its exponential map $\exp _{u}: \mathbf{R} \rightarrow D_{u}$ given by $\exp _{u}(\lambda)[x]=x+\left(e^{\lambda}-1\right)\langle x, u\rangle u$. The exponential map $\exp _{T}$ for the translation group of $\mathbb{R}^{d}$ can be described by $\exp _{T}(t)[x]=x+t, x, t$ in $\mathbf{R}^{d}$.

If there are elements $M \in S, t \in \mathbf{R}^{d}$, satisfying (6), we associate to every $x \in \mathbf{R}^{d}$ an orbit curve $\phi_{x}: \mathbf{R} \rightarrow \mathbf{R}^{d}$ by setting $\phi_{x}(\tau)=\exp _{T}(\tau t) \circ$ $\exp _{0}(\tau M) \circ \exp _{u}(-\tau)[x]$. Consider the set $\Pi=\{(x, n): x$ is a vertex of $P, n$ is the outer normal of $P$ at some facet containing $x\}$. Since $\Pi$ is finite, we use (6) 
and the easily established expression $\phi_{x}^{\prime}(0)=M x+t-\langle x, u\rangle u$ for the derivative of $\phi_{x}$, to obtain a number $\varepsilon>0$ such that $\left\langle\phi_{x}(\tau)-x, n\right\rangle<0$ for every $(x, n) \in \Pi$ and every $\tau \in] 0, \varepsilon\left[\right.$. If $\varepsilon$ is small enough, the additional inequalities $\left\langle\phi_{x}(\tau)-\right.$ $y, m\rangle<0$, for every vertex $x$ and every $(y, m) \in \Pi, y \neq x$, are automatically satisfied, which means $\phi_{x}(\tau) \in P$, for every vertex $x$ of $P$ and every $\tau \in[0, \varepsilon[$. Setting $r_{\tau}=\exp _{T}(\tau t) \circ \exp _{0}(\tau M) \in$ isom $_{+}\left(\mathbf{R}^{d}\right)$, we obtain $D\left(u, e^{-\tau}\right)[P] \subset r_{\tau}^{-1}[P]$, $\tau \in[0, \varepsilon[$, which is the claim of Lemma 5 .

A similar result is true for arbitrary convex bodies, but since it would require some extra work without giving, at the moment, any extra benefit, we omit it here.

Lemma 6. Let $P$ be a d-dimensional convex polytope in $\mathbf{R}^{d}$, such that the origin o of $\mathbb{R}^{d}$ is a local center for $P$. Then we can associate, to every $u \in S^{d-1}$, a skew symmetric $(d \times d)$-matrix $M$, satisfying

$$
\langle M x-\langle x, u\rangle u, n\rangle \leq 0
$$

for every vertex $x$ of $P$ and every outer normal $n$ of $P$ at $x$.

Proof. Consider, as in the proof of Lemma 5, for a fixed $u \in S^{d-1}$, the exponential maps $\exp _{0}: S \rightarrow \mathrm{O}(d)$ and $\exp _{u}: \mathbf{R} \rightarrow D_{u}$. Let $\left(\tau_{i}\right)_{i \in \mathbb{N}}$ be a sequence in 10,1$]$ converging to 0 . According to Definition 4 we obtain a sequence $\left(A_{i}\right)_{i \in \mathrm{N}}$ in $S$, converging to the zero matrix, such that $\exp _{0}\left(A_{i}\right) \exp _{u}\left(-\tau_{i}\right)[P] \subset P$, for sufficiently large $i$. Set $M_{i}=\left(1 / \tau_{i}\right) A_{i}$. If $\left(M_{i}\right)_{i \in \mathrm{N}}$ is a bounded sequence in the vector space $S$ of all skew symmetric $(d \times d)$-matrices, we may assume that it converges to some $M \in S$. For every vertex $x$ of $P$ and outer normal $n$ of $P$ at $x$ we have, for $i$ large enough, $\left\langle\exp _{0}\left(A_{i}\right) \circ \exp _{u}\left(-\tau_{i}\right)[x]-x, n\right\rangle \leq 0$, hence $0 \geq$ $\lim _{i \rightarrow \infty}\left\langle\left(1 / \tau_{i}\right)\left(\exp _{0}\left(A_{i}\right) \circ \exp _{u}\left(-\tau_{i}\right)[x]-x\right), n\right\rangle=\langle M x-\langle x, u\rangle u, n\rangle$, as stated under (7).

Assuming now that $\left(M_{i}\right)_{i \in N}$ is not bounded, we shall reach a contradiction. Denote by $\rho_{i}=\left\|M_{i}\right\|$ the norm of $M_{i}$, and set $N_{i}=\left(1 / \rho_{i}\right) M_{i}$. Again we may assume that $\left(N_{i}\right)_{i \in N}$ converges, to a matrix $N \in S, N \neq 0$. As above we obtain the relation $\langle N x, n\rangle \leq 0$, for every vertex $x$ of $P$ and every outer normal $n$ of $P$ at $x$. This is impossible, though, as we see by induction on $d$. The case $d=1$ is obvious. For the inductive step, let us choose a vertex $x$ of $P$ such that $\|x\| \geq\|y\|$, for every $y \in P$. After replacing $P$ by $s P$ and $N$ by $s N s^{-1}$, for a properly chosen similarity $s=\lambda r, r \in \mathrm{SO}(d), \lambda>0$, we may assume $x=e_{d}, d \geq 2$. Since $N$ is skew symmetric, we have $\langle N x, x\rangle=0$, and by using appropriate normal vectors $n$ of $P$ at $x$, we conclude from this and the relations $\langle N x, n\rangle \leq 0$, that $N x=0$. It follows that the restriction $N \mid x^{\perp}$ maps $x^{\perp}=\mathbb{R}^{d-1}$ into itself. We apply the inductive assumption to $N \mid x^{\perp}$ and to the projection $\pi_{x}[P]$. Remember $N \neq 0$, hence $N \mid x^{\perp} \neq 0$. Lemma 6 follows.

As an illustration of Lemma 6 we show here that no regular octahedron can cover its contractions, in the sense of Definition 3. Otherwise the origin $a$ would be a local center of $P=\operatorname{conv}\left(\left\{e_{i}: i \in Z_{3}\right\} \cup\left\{-e_{i}: i \in \mathbb{Z}_{3}\right\}\right)$, see Lemma 4 . Here, by abuse of language, we identify the set $\{1,2,3\} \subset \mathbf{Z}$ with the cyclic group $\mathbf{Z}_{3}$ of order three. Consider the pairs $\left(x_{i}, n_{i}\right), i \in \mathbf{Z}_{3}$, of vertices and outer normals of the polytope $P$, given by $x_{i}=e_{i}, n_{i}=(1 / \sqrt{3})\left(e_{i}-e_{i+1}-e_{i+2}\right)$. Applying the condition (7) of Lemma 6 to $u=(1 / \sqrt{3})(1,1,1)$ and to the pairs $\left(x_{i}, n_{i}\right)$, we 
would have to find a skew symmetric $(3 \times 3)$-matrix $M=\left(M_{j k}\right)$, satisfying $M_{12}-$ $M_{31}+1 / 3 \leq 0, M_{23}-M_{12}+1 / 3 \leq 0, M_{31}-M_{23}+1 / 3 \leq 0$, which is impossible. It would be interesting to use Lemma 6 for a direct proof of Theorem 3 below, and to look for analogues in connection with arbitrary convex bodies and their contractions.

\section{Polygons}

This section contains the main step toward our proof of the fact that every convex disc can cover its contractions. We identify $\mathbf{R}^{2}$ with the field $\mathbf{C}$ of complex numbers, and take advantage of its multiplicative structure. The imaginary unit in C is the vector $i=e_{2}=(0,1)$, so that multiplication by $i$ is a rotation with angle $\pi / 2$.

Definition 6. If $P \subset \mathbf{R}^{2}$ is a convex polygon, we denote by $\Delta^{0} P$ the set of its vertices and by $\Delta^{1} P$ the set of its edges. $n(P, k)$ stands for the outer normal of $P$ at $k \in \Delta^{1} P$. We set $\Pi(P)=\left\{(a, n(P, k)): a \in \Delta^{0} P, k \in \Delta^{1} P, a \in k\right\} . P$ is called nondegenerate, if no two of the lines $\operatorname{lin}\left\{e_{1}\right\}, \operatorname{lin}\left\{e_{2}\right\}, \operatorname{aff}(X)$, where $X$ runs through the two-point subsets of $\Delta^{0} P$, are parallel.

Definition 7. Given $a \in \mathbf{R}^{2}, n \in \mathbf{R}^{2} \backslash\{0\}$ and $\xi \in \mathbf{R}$, we consider the linear transformation $N_{\xi}: \mathbf{R}^{2} \rightarrow \mathbf{R}^{2}$, the open half-space $H(a, n) \subset \mathbf{R}^{2} \times \mathbf{R}=\mathbb{R}^{3}$ and the open half-plane $H_{\xi}(a, n) \subset \mathbf{R}^{2}$, defined by

$$
\begin{aligned}
N_{\xi} & =\pi_{e_{2}}-\xi i, \quad \text { or equally } \quad N_{\xi}=\left(\begin{array}{cc}
0 & \xi \\
-\xi & 1
\end{array}\right), \\
H(a, n) & =\left\{(x, \zeta) \in \mathbf{R}^{2} \times \mathbf{R}:\left\langle x-N_{\zeta}(a), n\right\rangle<0\right\}, \\
H_{\xi}(a, n) & =\left\{x \in \mathbf{R}^{2}:(x, \xi) \in H(a, n)\right\} .
\end{aligned}
$$

Lemma 7. With the notation of Definition 7 we have

$H_{\xi}(a, n) \cap H_{\xi}(b, n) \subset H_{\xi}(\lambda a+\mu b, n)$, if $\lambda>0, \mu>0$, and $\lambda+\mu=1$.

$H_{\xi}(a, m) \cap H_{\xi}(a, n) \subset H_{\xi}(a, \lambda m+\mu n)$, if $m, n$ are linearly independent and $\lambda>0, \mu>0$. Furthermore, $H_{\xi}(a, m) \cap H_{\xi}(a, n)$ is the open convex cone with apex $N_{\xi}(a)$ and outer normals $m, n$.

Also, consider a finite set $\mathscr{H}$ of open half-spaces $H(a, n), a \in \mathbb{R}^{2}, n \in \mathbb{R}^{2} \backslash\{o\}, a$ vector $t \in \mathbf{R}^{2}$ and a reflection $\sigma: \mathbb{R}^{2} \rightarrow \mathbb{R}^{2}$ at one of the axes $\operatorname{lin}\left\{e_{1}\right\}, \operatorname{lin}\left\{e_{2}\right\}$. Then

the intersection $\cap \mathscr{H}$ of the half-spaces in $\mathscr{H}$ is not empty, provided that

$\cap \mathscr{L} \neq \varnothing$, for every subset $\mathscr{L}$ of $\mathscr{H}$ with at most four elements;

$\cap \mathscr{H} \neq \varnothing$ if, and only if

$\bigcap\{H(a+t, n): H(a, n) \in \mathscr{H}\} \neq \varnothing$, or

$\bigcap\{H(\sigma a, \sigma n): H(a, n) \in \mathscr{H}\} \neq \varnothing$. 
Proof. In order to see (10) we apply Helly's theorem [2] to the set $\mathscr{H}$ of open half-spaces in $\mathbb{R}^{3}$. The remaining statements follow from easy exercises in linear algebra.

Definition 8. A convex polygon $P \subset \mathbf{R}^{2}$ is good, if

$$
\bigcap\{H(a, n):(a, n) \in \Pi(P)\} \neq \varnothing .
$$

If we remember that $i$ is the imaginary unit in $\mathbb{R}^{2}=\mathbf{C}$ and compare (12) with (6) in Lemma 5, we see that a good convex polygon can cover its small contractions in direction $e_{2}$.

Definition 9. A standard representation of the convex $r$-gon $P \subset \mathbb{R}^{2}$ is a bijective map $k \mapsto a_{k}$ from the cyclic group $\mathbf{Z}_{r}$ with $r$ elements onto $\Delta^{0} P$, such that $l_{k}=\operatorname{conv}\left\{a_{k+1}, a_{k}\right\} \in \Delta^{1} P$, for every $k$. Setting $n_{k}=n\left(P, l_{k}\right)$ according to Definition 6, we introduce the sets $A_{\xi}^{k}=H_{\xi}\left(a_{k}, n_{k}\right) \cap H_{\xi}\left(a_{k}, n_{k-1}\right)$, and the angular polyhedron $A(P)=\bigcap\left\{H\left(a_{k}, n_{k}\right) \cap H\left(a_{k}, n_{k-1}\right): k \in \mathbb{Z}_{r}\right\}$.

Lemma 8. Every nondegenerate triangle $D \subset \mathbf{R}^{2}$ is good.

Proof. With the notation of Definition 9, we consider a standard representation $k \mapsto a_{k}, k \in \mathbf{Z}_{3}=\{0,1,2\}$, of $D$. We have to show $A(D) \neq \varnothing$, or equivalently,

$$
\bigcap\left\{A_{\xi}^{k}: k \in \mathbb{Z}_{3}\right\} \neq \varnothing, \text { for some } \xi \in \mathbf{R} \text {. }
$$

Let $\arg (x) \in\left[0,2 \pi\left[\right.\right.$ be the argument of $x \in \mathbf{C} \backslash\{0\}$, such that $x=\|x\| e^{t \arg (x)}$. After appropriate reflections at the axes $\operatorname{lin}\left\{e_{1}\right\}, \operatorname{lin}\left\{e_{2}\right\}$ and by changing the representation $k \mapsto a_{k}$, if needed, we may assume, in view of (11),

$$
\pi / 2<\nu_{1}<\nu_{0}-\pi<\nu_{2}<3 \pi / 2<\nu_{0}<2 \pi,
$$

where $\nu_{k}=\arg \left(n_{k}\right), k \in \mathbf{Z}_{3}$. We study three cases, (15), (16), and (17).

$$
\nu_{2}<\pi \text {. }
$$

We use (11) to obtain $o \in \operatorname{aff}\left\{a_{0}, a_{1}\right\} \cap\left(a_{2}+\operatorname{lin}\left\{e_{1}\right\}\right)$, after a translation of $D$. Setting $\alpha_{k}=\arg \left(a_{k}\right), k \in \mathbf{Z}_{3}$, and $\xi_{0}=\sin \alpha_{0} \cos \alpha_{0}=\sin \alpha_{1} \cos \alpha_{1}$, we find $N_{\xi_{0}}\left(a_{k}\right)$ $=\left(\sin ^{2} \alpha_{0}\right) a_{k}, k \in\{0,1\}$, so it follows from (9) and Definition 7 that $o \in \operatorname{cl}\left(A_{\xi_{0}}^{0} \cap\right.$ $\left.A_{\xi_{0}}^{1}\right)$. Now we use the remark, that $o \in H_{\xi}(a, n)$, for every $a \in \mathbb{R}^{2} \backslash\{o\}, n \in \mathbb{R}^{2} \backslash$ $\{o\}$, is equivalent to $\left\langle N_{\xi}(a), n\right\rangle>0$, where $\left\langle N_{\xi}(a), n\right\rangle=a_{2} n_{2}-\left(a_{1} n_{2}-a_{2} n_{1}\right)=$ $\|a\|\|n\|(\sin \alpha \sin \nu-\xi \sin (\nu-\alpha))$ with $\alpha=\arg (a), \nu=\arg (n)$. This, together with (14) and (15), immediately implies $o \in A_{\xi_{0}}^{2}$, and (13) is established in this case.

$$
\nu_{2}>\pi \text { and } 0<\nu_{0}-\nu_{2} \leq \pi / 2 \text {. }
$$

This time we assume $a_{2}=0$ and obtain in the same way as above for $\xi_{1}=$ $\sin \alpha_{1} \cos \alpha_{1}$ the relations $o \in \operatorname{cl}\left(A_{\xi_{1}}^{1} \cap A_{\xi_{1}}^{2}\right)$ and $o \in A_{\xi_{1}}^{0}$.

$$
\nu_{2}>\pi \text { and } \pi / 2<\nu_{0}-\nu_{2}<\pi \text {. }
$$


Assume $a_{2}=o$. It follows that $o \in \operatorname{cl}\left(A_{\xi}^{2}\right)$, for every $\xi \in \mathbb{R}$. Set $\delta=\nu_{0}-(3 \pi / 2)$, $\xi_{1}=\sin \alpha_{1} \cos \delta / \cos \left(\delta-\alpha_{1}\right), \quad \xi_{2}=\sin \alpha_{1} \cos \alpha_{1}, \quad \xi_{3}=\sin \alpha_{0} \cos \alpha_{0}$, and $\xi_{4}=$ $\sin \alpha_{0} \cos \delta / \cos \left(\delta-\alpha_{0}\right)$. Then $0<\xi_{1}<\xi_{2}$, because the function $f$, given by $f(t)=$ $\sin \alpha_{1} \cos t / \cos \left(\alpha_{1}-t\right)$ is strictly decreasing and continuous on $] \alpha_{1}-(\pi / 2), \alpha_{1}+$ $(\pi / 2)\left[\right.$, with $0=f(\pi / 2), \xi_{1}=f(\delta), \xi_{2}=f\left(\alpha_{1}\right)$; note that we use (14). Similarly we establish $\xi_{3}<\xi_{4}$. Using the remark above we find $o \in A_{\xi}^{1}$ for $\left.\xi \in\right] \xi_{1}, \xi_{2}[$ and $o \in A_{\xi}^{0}$ for $\left.\xi \in\right] \xi_{3}, \xi_{4}\left[\right.$. Now $\xi_{3}<0<\xi_{1}$ and $\xi_{1}<\xi_{4}$. This last inequality is due to the fact that the function $g$, given by $g(t)=\cos \delta \sin t / \cos (\delta-t)$ is strictly increasing and continuous on $] \delta-(\pi / 2), \delta+(\pi / 2)\left[\right.$, with $\xi_{1}=g\left(\alpha_{1}\right), \xi_{4}=g\left(\alpha_{0}-\right.$ $\pi),-\pi / 2<\delta-\left(\alpha_{0}-\pi\right)<0<\delta-\alpha_{1}<\pi / 2$. Note that we use (17). As a consequence, $] \xi_{1}, \xi_{2}[\cap] \xi_{3}, \xi_{4}[\neq \varnothing$, and (13) follows in this case, too. Lemma 8 is established.

Lemma 9. Every nondegenerate quadrangle $Q \subset \mathbf{R}^{2}$ is good.

Proof. In view of (12) and (10) we have to show

$$
\bigcap_{k=0}^{3} H\left(a_{k}, n_{k}\right) \neq \varnothing,
$$

for every set $\left\{\left(a_{k}, n_{k}\right): 0 \leq k \leq 3\right\} \subset \Pi(Q)$ with four elements. Again we consider three cases.

$$
\text { Assume }\left\{a_{k}: 0 \leq k \leq 3\right\} \neq \Delta^{0} Q
$$

We choose $x \in \Delta^{0} Q \backslash\left\{a_{k}: 0 \leq k \leq 3\right\}$ and set $D=\operatorname{conv}\left(\Delta^{0} Q \backslash\{x\}\right)$. Lemma 8 , applied to $D$, and the relation (9) of Lemma 7 immediately imply $\cap_{k=0}^{3} H\left(a_{k}, n_{k}\right)$ $\supset A(D) \neq \varnothing$.

$$
\text { Assume }\left\{n_{k}: 0 \leq k \leq 3\right\} \neq\left\{n(Q, l): l \in \Delta^{1} Q\right\} \text {. }
$$

If $o \notin \operatorname{conv}\left\{n_{k}: 0 \leq k \leq 3\right\}$ then, by the nondegeneracy of $Q$, the vectors $n_{k}$ lie all in some open semicircle on $S^{1}$ and $\cap_{k=0}^{3} H_{\xi}\left(a_{k}, n_{k}\right)$ is a nonvoid, open, unbounded convex set in $\mathbf{R}^{2}$, for every $\xi \in \mathbf{R}$. In the case $o \in \operatorname{conv}\left\{n_{k}: 0 \leq k \leq 3\right\}$ the set $D=\left\{x \in \mathbb{R}^{2}:\left\langle x, n_{k}\right\rangle \leq\left\langle a_{k}, n_{k}\right\rangle\right\}$ is a nondegenerate triangle, and Lemma 8 , together with $(8)$, again produce $\bigcap_{k=0}^{3} H\left(a_{k}, ; n_{k}\right) \supset A(D) \neq \varnothing$. So we are left with

$$
\left\{a_{k}: 0 \leq k \leq 3\right\}=\Delta^{0} Q, \quad\left\{n_{k}: 0 \leq k \leq 3\right\}=\left\{n(Q, l): l \in \Delta^{\mathrm{l}} Q\right\} .
$$

Using a reflection at $\operatorname{lin}\left\{e_{1}\right\}$, if needed, we may assume, in view of (11), that $k \mapsto a_{k}, k \in \mathbb{Z}_{\mathbf{4}}=\{0,1,2,3\}$, is a standard representation of $Q$, and that $n_{k}=$ $-i\left(a_{k+1}-a_{k}\right) /\left\|a_{k+1}-a_{k}\right\|$, for every $k$. If there exists a point $b \in \mathbb{R}^{2}$ such that $\left\langle i\left(a_{k}-b\right), n_{k}\right\rangle>0$, for each $k \in \mathbb{Z}_{4}$, we may assume, by (11), that $b$ is the origin $o$. Then, for every $\xi<0$ with $|\xi|$ sufficiently large, we obtain $o \in \bigcap_{k=0}^{3} H_{\xi}\left(a_{k}, n_{k}\right)$, and (18) is satisfied. If we find no point $b$ such that $\left\langle i\left(a_{k}-b\right), n_{k}\right\rangle>0$ for each $k \in \mathbb{Z}_{4}$, then the half-planes $E_{k}=\left\{x \in \mathbb{R}^{2}:\left\langle x-a_{k}, i n_{k}\right\rangle>0\right\}, k \in \mathbb{Z}_{4}$, have 
empty intersection so, by Helly's theorem, some triplet, say $E_{0}, E_{1}, E_{2}$, is without a common point, too. Considering that $a_{k+1} \in E_{k}$, we see that the triangle $N_{1}=\operatorname{conv}\left\{a_{0}, a_{1}, a_{2}\right\}$ has an obtuse angle at $a_{0}$, and $N_{2}=\operatorname{conv}\left\{a_{0}, a_{3}, a_{2}\right\}$ has an obtuse angle at $a_{2}$. Because of (11) we may assume $o \in \operatorname{aff}\left\{a_{0}, a_{2}\right\} \cap$ $\operatorname{aff}\left\{a_{1}, a_{3}\right\}$. Set $t_{k}=\operatorname{aff}\left\{a_{1}, a_{3}\right\} \cap\left(a_{k}+\operatorname{lin}\left\{n_{k}\right\}\right), k \in\{0,2\}$. Since the angles at $a_{0}$ and $a_{2}$ are obtuse, we find real numbers $\lambda_{1}$, with $\lambda_{0}<\lambda_{1}<0<\lambda_{2}<\lambda_{3}$ such that $a_{1}=\lambda_{0} a, t_{0}=\lambda_{1} a, t_{2}=\lambda_{2} a, a_{3}=\lambda_{3} a$, where $a=\left(1 /\left\|a_{3}\right\|\right) a_{3}$. Set $\alpha=$ $\arg (a) \in\left[0,2 \pi\left[\right.\right.$, such that $a=e^{i \alpha}$, and $\xi=\sin \alpha \cos \alpha$. Then $\xi \neq 0$ and $N_{\xi}(\lambda a)=$ $\left(\lambda \sin ^{2} \alpha\right) a \neq o$, for every $\lambda \neq 0$, by the nondegeneracy of $Q$. This says $N_{\xi}\left(t_{k}\right) \in$ rel int conv $\left\{o, a_{k+1}\right\}$ for $k \in\{0,2\}$, and consequently $o \in \bigcap_{k \in\{0,2\}}\left(H_{\xi}\left(t_{k}, n_{k}\right) \cap\right.$ $\left.H_{\xi}\left(t_{k}, n_{k+1}\right)\right)$. A straightforward computation gives $N_{\xi}\left(t_{k}\right) \in H_{\xi}\left(a_{k}, n_{k}\right) \cap$ $H_{\xi}\left(a_{k+1}, n_{k+1}\right), k \in\{0,2\}$. Combining this with (9), we obtain $H_{\xi}\left(t_{k}, n_{k}\right) \cap$ $H_{\xi}\left(t_{k}, n_{k+1}\right) \subset H_{\xi}\left(a_{k}, n_{k}\right) \cap H_{\xi}\left(a_{k+1}, n_{k+1}\right)$. We have now reached

$$
o \in \bigcap_{k \in\{0,2\}}\left(H_{\xi}\left(t_{k}, n_{k}\right) \cap H_{\xi}\left(t_{k}, n_{k+1}\right)\right) \subset \bigcap_{k \in \mathbf{Z}_{3}} H_{\xi}\left(a_{k}, n_{k}\right),
$$

so that (18) follows in this case, too. Our proof of Lemma 9 is completed.

Lemma 10. Every nondegenerate convex polygon $P \subset \mathbf{R}^{2}$ is good.

Proof. Remembering (10) and (12) we have to show

$$
\bigcap_{k=0}^{3} H\left(a_{k}, n_{k}\right) \neq \varnothing,
$$

for every set $\left\{\left(a_{k}, n_{k}\right): 0 \leq k \leq 3\right\} \subset \Pi(Q)$ with four elements. Let us choose $X \subset \Delta^{0} Q$ with card $X \in\{3,4\}$ and $\left\{a_{k}: 0 \leq k \leq 3\right\} \subset X . Q=\operatorname{conv}(X)$ is a nondegenerate triangle or quadrangle, and Lemmas 8 and 9 , together with (9), imply $\bigcap_{k=0}^{3} H\left(a_{k}, n_{k}\right) \supset A(Q) \neq \varnothing$, as required for the proof of Lemma 10.

\section{Local Centers and Local Axes}

The material of this section will be used to show that covering one's contractions is not easy, in higher dimensions.

Definition 10. Let $P \subset \mathbf{R}^{d}$ be a convex polytope, and $z$ a point of $\operatorname{relint}(P)$. $F \in \partial P$ is called an exposed face of $P$, with respect to $z$, if there exists some $q \in \operatorname{relint}(F)$ such that $(q-z) /\|q-z\|$ is an outer normal of $P$ at $F$.

Note that, under those circumstances, the nearest point map $\nu_{F}$ carries $z$ to $q$.

Lemma 11. For each $z$ in the relative interior of the convex polytope $P \subset \mathbf{R}^{d}$, each vertex of $P$ with maximal distance from $z$ and each facet $F$ of $P$ with minimal distance from $z$ are exposed faces of $P$, with respect to $z$.

The proof of Lemma 11 is obvious, and shall be omitted here. 
Lemma 12. Let $P \subset \mathbb{R}^{d}$ be a convex d-polytope, with $o \in \operatorname{int}(P)$. If the origin $o$ is a local center for $P$, and $F \in \partial P$ is exposed for $P$, with respect to $o$, then $\operatorname{lin}\left\{\nu_{F}(o)\right\}$ is a local axis for $P$.

Proof. This is clear in the case $\operatorname{dim}(F)=0$. If $\operatorname{dim}(F)=d-1$, set $S=\operatorname{aff}(F)$, $q=\nu_{F}(o), \delta=\left(\frac{1}{2}\right) d(q, \operatorname{relbd}(F))$, and choose a neighbourhood $N$ of 1 in $\operatorname{SO}(d)$ such that $d(q, S \cap r S)<\delta$, for every $r \in N$. Since $o$ is a local center for $P$, we can associate, to every $u \in S^{d-1} \cap q^{\perp}$ and $\left.\left.\lambda \in\right] 0,1\right]$ sufficiently close to 1 , a rotation $r \in N$ satisfying $D(u, \lambda)[P] \subset r P$. But, for $\lambda$ close enough to 1 , the set $D(u, \lambda)[F]$ contains the $(d-1)$-dimensional ball $B=\{x \in S ;\|x-q\| \leq \delta\}$. In the case $r(q) \neq q$ we have $r S \cap B \neq \varnothing$, and since $r S$ is a supporting hyperplane of $r P$, this implies $B \not \subset r P$, and consequently $D(u, \lambda)[P] \not \subset r P$. It follows that $\operatorname{lin}\{q\}$ is indeed a local axis for $P$. A similar argument works for $0<\operatorname{dim}(F)<d-1$; we omit the details, and will not use the corresponding result.

Definition 11. Let $\mathscr{S}$ be a set of convex polygons in $\mathbb{R}^{2}$. The origin $o$ is a local center for $\mathscr{S}$, if $o \in \operatorname{int}(P)$, for every $P \in \mathscr{S}$, and, furthermore, given any $u \in S^{1}$ and any neighborhood $N$ of the identity in $\mathrm{SO}(2)$, we can find a number $\delta \in] 0,1[$ and a map $\lambda \mapsto r_{\lambda}$ from $[1-\delta, 1]$ into $N$, such that $D(u, \lambda)[P] \subset r_{\lambda} P$, for every $P \in \mathscr{S}$ and every $\lambda \in[1-\delta, 1]$.

This means that a small contraction in any direction $u$ can be covered by a small rotation, simultaneously, for all members of $\mathscr{S}$.

Lemma 13. Consider a set $\mathscr{P}$ of convex polygons in $\mathbb{R}^{2}$, such that the origin o is a local center for $\mathscr{S}$. Let $k$ be an exposed edge for some $P \in \mathscr{S}$, with respect to o. Given any $Q \in \mathscr{S}$, denote by $L_{1}, L_{2}$ the supporting lines of $Q$ which are parallel to $\operatorname{aff}(k)$, and set $l_{i}=Q \cap L_{i}$. Then $l_{1}$ and $l_{2}$ are always exposed edges of $Q$, with respect to $o$.

Proof. Otherwise we may assume that there are polygons $P, Q$ in $\mathscr{S}$ such that $k=\left\{x \in P: x_{1} \leq y_{1}\right.$ for every $\left.y \in P\right\}$ is an exposed edge for $P$, with respect to $o$, whereas $l=\left\{x \in Q: x_{1} \geq y_{1}\right.$ for every $\left.y \in Q\right\}$ is not an exposed edge of $Q$, with respect to $o$. Therefore we have, without lack of generality, $x_{2} \geq 0$, for every $x \in l$. Determine the vertices $v \in k \cap \Delta^{0} P, w \in l \cap \Delta^{0} Q$ by requiring $v_{2} \geq x_{2}$, for every $x \in k, w_{2} \leq y_{2}$, for every $y \in l$. Clearly $v_{2}>0, w_{2} \geq 0$. The direction $-e_{1}$ is an outer normal of $P$ at $v$, satisfying $\left\langle i v,-e_{1}\right\rangle>0$, and by the construction of $w$, we find an outer normal $n$ of $Q$ at $w$, such that $\langle i w, n\rangle<0$. Let us choose a small number $\tau>0$, and set $u=(\sin \tau, \cos \tau)$. The condition (7), with $M=\left(\begin{array}{cc}0 & -\xi \\ \xi & 0\end{array}\right)$, applied to $u$ and $\left(P, v,-e_{1}\right)$ and to $(Q, w, n)$ leads, after a short calculation, to the inequalities $\xi\left\langle i v,-e_{1}\right\rangle+\langle v, u\rangle\left\langle u, e_{1}\right\rangle \leq 0$ and $\xi\langle i w, n\rangle-\langle w, u\rangle\langle u, n\rangle \leq 0$, which have to be fulfilled simultaneously by some $\xi \in \mathbb{R}$. If we choose $\tau$ sufficiently small, we obtain a contradiction between these inequalities. Thus Lemma 13 is established. 


\section{Some Results}

Theorem 1. Every two-dimensional compact convex set can cover its contractions.

Proof. Denote by $\mathscr{P}$ the set of all convex polygons $P \subset \mathbb{R}^{2}$ which are nondegenerate, in the sense of Definition 6. We derive from Lemma 10, Definition 8, and Lemma 5 , that every $P \in \mathscr{P}$ can cover its small contractions in direction $e_{2}$. The statement (4) in Lemma 2 shows that every $P \in \mathscr{P}$ can cover its contractions in direction $e_{2}$. Since $\mathscr{P}$ is dense in the set $\mathscr{C}_{2}$, we use (2) to conclude that every two-disc $C \in \mathscr{C}_{2}$ can cover its contractions in direction $e_{2}$. In order to complete our proof of Theorem 1 , we just apply (5) to the class $\mathscr{C}_{2}$.

The situation in higher dimensions is not clear to the authors of this essay, as the two following theorems illustrate.

Theorem 2. Consider a convex set $C \in \mathscr{C}_{d}, d \geq 3$. If either $\operatorname{dim}(C) \leq 2$ or else $C$ is a $k$-dimensional ellipsoid for some $k \leq d$, then every parallel set $C+\rho B^{d}, \rho \geq 0$, can cover its contractions.

Proof. In view of Theorem 1 and Lemma 3 it remains to show that every ellipsoid $C$ can cover its contractions. To this end we may assume $C=\left\{x \in \mathbb{R}^{k}\right.$ : $\left.\sum_{i=1}^{k}\left(x_{i} / \alpha_{i}\right)^{2} \leq 1\right\}, \alpha_{1} \geq \alpha_{2} \geq \cdots \geq \alpha_{k}>0$. A well known minimax principle (see, for example [7], p. 263) gives the relations $\alpha_{i}=\min \left\{\max _{x \in C \cap E}\|x\|: E \in\right.$ $\Gamma(k, k-i+1)\}$. If $f: \mathbb{R}^{k} \rightarrow \mathbf{R}^{k}$ is a nondegenerate contraction, we set $D=f[C]$ and $\beta_{i}=\min \left\{\max _{x \in D \cap E}\|x\|: E \in \Gamma(k, k-i+1)\right\}$. Obviously $\beta_{i} \leq \alpha_{i}, 1 \leq i \leq k$. Since $D$ is congruent to the ellipsoid $D_{0}=\left\{x \in \mathbf{R}^{k}: \sum_{i=1}^{k}\left(x_{i} / \beta_{i}\right)^{2} \leq 1\right\} \subset C$, it can be covered by $C$, and Theorem 2 follows.

Notice that each set $A \in \mathscr{C}_{d}$, as described by the above theorem, can also cover its shadows in $\mathbf{R}^{n}, n \geq d$. We just have to apply (3) of Lemma 2 . The corresponding statement is not true for any polytope.

Theorem 3. No d-dimensional convex polytope can cover its contractions, provided that $d \geq 3$.

Proof. If there is a counterexample, then Lemma 4 also guarantees the existence of a $d$-polytope $P \subset \mathbf{R}^{d}$ such that

the origin $o$ of $\mathbb{R}^{d}$ is a center of symmetry and, at the same time, a local center for $P$.

We are going to show, by induction on $d$, that no such polytope $P$ with $d=\operatorname{dim}(P) \geq 3$ can occur. Consider first a three-polytope $P \subset \mathbb{R}^{3}$, satisfying (23). Lemmas 11 and 12 say that any vertex $p$ of $P$ with maximal norm $\|p\|$ is an exposed face of $P$, with respect to $o$, and that $\operatorname{lin}\{p\}$ is a local axis. An appropriate similarity transformation allows us to assume $p=e_{3}$, and we set, for $\lambda \in]-1,1\left[, P_{\lambda}=\pi_{p}\left[P \cap\left(p^{1}+\lambda p\right)\right] \subset p^{1}=\mathbf{R}^{2}\right.$. Comparing Definition 5 with Definition 11, we notice that $o$ is a local center for the set $\mathscr{S}=\left\{P_{\lambda^{*}}: \lambda \in\right]-1,1[\}$. 
According to Lemma 11, some edge $k$ of $P_{0}=P \cap \mathbf{R}^{2}$ is an exposed face of $P_{0}$, and Lemma 13 implies the existence of two exposed edges parallel to $k$, with respect to $o$, for every $\left.P_{\lambda}, \lambda \in\right]-1,1[$. Choosing a unit vector $u$ such that $\operatorname{lin}\{u\}$ and $\operatorname{aff}(k)$ are parallel, we conclude that $\pi_{u}(P)=P \cap u^{\perp}$, and that, for each point $q$ in $\left(\operatorname{relbd} \pi_{u}(P)\right) \backslash\{p,-p\}$, the line $q+\operatorname{lin}\{u\}$ intersects $P$ in a line segment $S_{q} \subset \operatorname{bd}(P)$, which has positive length and contains $q$ in its relative interior. On the other hand,

$$
S_{x}=P \cap(x+\operatorname{lin}\{u\})=\{x\}, \text { for } x \in\{p,-p\} .
$$

The set $\operatorname{SB}(P, u)=\bigcup\left\{S_{x}: \quad x \in \operatorname{relbd} \pi_{u}(P)\right\}=P \cap \pi_{u}^{-1}\left[\operatorname{relbd} \pi_{u}(P)\right]$ is usually called the shadow boundary of $P$ in direction $u$, and (24) states that $\operatorname{SB}(P, u)$ is pinched at $p$ and at $-p$. By Lemma 11 the polygon $\pi_{u}(P)$ has an edge $l$ which is an exposed face of $\pi_{u}(P)$, with respect to $o$; hence the facet $F=\bigcup\left\{S_{x}: x \in l\right\} \in \partial P$ is exposed for $P$, with respect to $o$. It follows that the nearest point maps $\nu_{l}, \nu_{F}$ satisfy $\nu_{l}(o)=\nu_{F}(o)=\bar{p} \in \operatorname{relint}(F)$, and that for at least one endpoint of $l$, say $r$, the set $S_{r}$ is an edge of $F$, and is exposed for $F$, with respect to $\bar{p}$. We set, for $\lambda \in[-1,1], \bar{P}_{\lambda}=\pi_{(\bar{p} /\|\bar{p}\|)}\left(P \cap\left(\bar{p}^{\perp}+\lambda \bar{p}\right)\right)$, and $\overline{\mathscr{S}}=\left\{\bar{P}_{\lambda}: \lambda \in[-1,1]\right\}$. Notice that $\bar{P}_{1}=\pi_{(\bar{p} /\|\bar{p}\|)}(F)$; hence $\pi_{(\bar{p} /\|\tilde{p}\|)}\left(S_{r}\right)$ is exposed for $\bar{P}_{1}$, with respect to $o$, and parallel to $u$. Just as above it follows that the plane section $P \cap\left(\bar{p}^{\perp}+\lambda \bar{p}\right)$ has two edges parallel to $u$, but this time for every $\lambda$ in the closed interval $[-1,1]$. Taking also $F$ and $-F$ into account, we see that $S_{x}$ is a nondegenerate line segment, containing $x$ in its relative interior, for every $x \in \operatorname{relbd} \pi_{u}(P)$. In other words, the shadow boundary $\operatorname{SB}(P, u)$ is everywhere broad, contradicting (24). Thus Theorem 3 is established in the case $d=3$. For the inductive step, assume that the $d$-polytope $P \subset R^{d}$ satisfies (23), for $d \geq 4$. We choose a vertex $x$ of $P$ with maximal norm $\|x\|$, and there is no loss of generality if we set $x=e_{d}$. By Lemma $12, \operatorname{lin}\{x\}$ is a local axis for $P$; hence the origin $o$ of $x^{\perp}=\mathbf{R}^{d-1}$ is a center of symmetry, as well as a local center, for $\pi_{x}[P]$, contradicting the inductive hypothesis. Theorem 3 follows.

\section{Questions}

In view of the preceding theorems, we feel that the following main problem may not have an easy solution.

Question 1. Which convex bodies can cover their contractions?

It would be interesting to know the answer for certain subclasses of $\mathscr{C}_{d}$, with appropriate regularity conditions.

In another direction, Gale [3] has constructed a general contraction $f: P \rightarrow Q$ from a rectangle $P$ of minimal width 1 onto a square $Q$ of minimal width 2 . With this in mind we ask:

Question 2. Are the Euclidean balls the only convex bodies in $\mathbb{R}^{d}$ which can cover all their general contractions $X \subset \mathbb{R}^{d}$ ?

There are several variants of this question. Let us say that $X \subset \mathbb{R}^{d}$ is a compression of $C \in \mathscr{C}_{d}$, if there exists a bijective general contraction from $C$ onto 
$X$. Remember that Gale's square $Q$ is not a compression of the rectangle $P$. Now we can try to determine those convex bodies $C \in \mathscr{C}_{d}$ which can cover all their compressions, or convex compressions, in $\mathbb{R}^{d}$. Similar problems may be posed in infinite dimensional, or curvilinear, spaces.

Question 3. Which polyhedra $P \subset \mathbb{R}^{d}$ can cover their contractions?

Here the term polyhedron means some finite union of convex polytopes. The analogue of Question 3 could be asked for arbitrary subsets of $\mathbb{R}^{d}$.

Question 4. Which convex bodies $C$ of $R^{d}$ can cover their shadows in $\mathbf{R}^{d}$ ?

Even for $d$-polytopes this question is not answered by Theorem 3. As an illustration we construct a class of sets in $\mathbb{R}^{3}$ which can cover their shadows. Consider any set $S$ in the unit ball $B^{3}$, satisfying $S=-S$. Choose a centrally symmetric convex disc $F=-F$ in $\mathbb{R}^{2}$, such that $B^{2} \subset F . C=\operatorname{clconv}(S \cup F)$ can cover its shadows. Namely, by Theorem 1 and (3), $F$ can cover $\pi_{u}(F)$, for every $u \in S^{2}$, and considering Lemma 4 , we may assume $\pi_{u}(F) \subset r(F)$, for some $r \in \mathrm{SO}(3)$ carrying $\mathbf{R}^{2}$ onto $u^{\perp}$. But $r(F) \subset u^{\perp}$ contains $B^{3} \cap u^{\perp}$, and therefore $\pi_{u}(S)$. It follows that $\pi_{u}(C)=\mathrm{clconv}\left(\pi_{u} S \cap \pi_{u} F\right) \subset r(F) \subset r(C)$. Similar constructions could obviously be carried out in higher dimensions. On the other hand the regular octahedron $P$, as described under Lemma 6, cannot cover all its shadows in $\mathbf{R}^{3}$. Actually $H=\pi_{u}(P), u=(1 / \sqrt{3})(1,1,1)$, cannot be covered by $P$. $H$ is a regular hexagon of side length $s=\sqrt{(2 / 3)}$. If $P$ could cover $H$, we would find, in view of Lemma 4, a rotation $r \in \mathrm{SO}(3)$, satisfying $L=r(H) \subset P$. But every point $x$ with $\|x\|=s$ has distance less than $\rho:=(s / 2)(\sqrt{3}-1)$ from some vertex $y$ of $P$. Since $s \geq 2 \rho$, each ball $B_{\rho}(y)=\left\{z \in \mathbf{R}^{3}:\|z-y\| \leq \rho\right\}, y \in \Delta^{0} P$, can contain at most one of the six vertices of $L$. Consequently $\Delta^{0} L \cap B_{\rho}(y) \neq \varnothing$, for every $y \in \Delta^{0} P$. But it is easy to see that no two-dimensional subspace of $\mathbb{R}^{3}$ can meet every ball $B_{\rho}(y), y \in \Delta^{0} P$. We have reached a contradiction, and $P$ cannot cover its shadows.

Question 5. Is there an efficient algorithm to decide whether a given $d$-polytope $P$ can cover the $d$-polytope $Q \subset \mathbb{R}^{d}, d \geq 3$ ?

\section{References}

1. C. Chevalley, Theory of Lie Groups, Vol. 1, Princeton University Press, Princeton, 1946

2. L. Danzer, B. Grünbaum, and V. Klee, Helly's theorem and its relatives, Convexity, Proc. Seventh Symp. Pure Math., Seattle, 1961, 101-180, Amer. Math. Soc., Providence, R.I., 1963.

3. D. Gale, On Lipschitzian mappings of convex bodies, Convexity, Proc. Seventh Symp. Pure Math., Seattle, 1961, 221-223, Amer. Math. Soc., Providence, R.I., 1963.

4. B. Grünbaum, Convex Polytopes, Wiley-Interscience, New York, 1967.

5. M. D. Kowaljov, Covering a convex figure by its images under dilatations (in Russian), Ukrainskij Geometriceskij Sbornik, 27/84 (1984), 57-68.

6. P. McMullen and G. C. Shephard, Convex Polytopes and the Upper Bound Conjecture, Cambridge University Press, 1971.

7. W. Nef, Lehrbuch der linearen Algebra, Birkhäuser, 1966.

8. J. Pach, Communication at the problem session, Tagung über konvexe Körper, Oberwolfach, 1984.

Received July 25, 1985. 